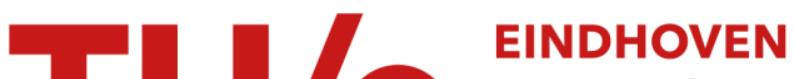 \\ UNIVERSITY OF \\ TECHNOLOGY
}

\section{On finite-time control of inner systems}

\section{Citation for published version (APA):}

Ludlage, J. H. A., Weiland, S., \& Stoorvogel, A. A. (1998). On finite-time control of inner systems. (Measurement and control systems : internal report; Vol. 98//04). Eindhoven University of Technology.

\section{Document status and date:}

Published: 01/01/1998

\section{Document Version:}

Publisher's PDF, also known as Version of Record (includes final page, issue and volume numbers)

\section{Please check the document version of this publication:}

- A submitted manuscript is the version of the article upon submission and before peer-review. There can be important differences between the submitted version and the official published version of record. People interested in the research are advised to contact the author for the final version of the publication, or visit the DOI to the publisher's website.

- The final author version and the galley proof are versions of the publication after peer review.

- The final published version features the final layout of the paper including the volume, issue and page numbers.

Link to publication

\section{General rights}

Copyright and moral rights for the publications made accessible in the public portal are retained by the authors and/or other copyright owners and it is a condition of accessing publications that users recognise and abide by the legal requirements associated with these rights.

- Users may download and print one copy of any publication from the public portal for the purpose of private study or research.

- You may not further distribute the material or use it for any profit-making activity or commercial gain

- You may freely distribute the URL identifying the publication in the public portal.

If the publication is distributed under the terms of Article $25 \mathrm{fa}$ of the Dutch Copyright Act, indicated by the "Taverne" license above, please follow below link for the End User Agreement:

www.tue.nl/taverne

\section{Take down policy}

If you believe that this document breaches copyright please contact us at:

openaccess@tue.nl

providing details and we will investigate your claim. 


\section{te}

Eindhoven University of Technology Department of Electrical Engineering

On Finite-Time Control of Inner Systems

Jobert Ludlage, Siep Weiland and Anton Stoorvogel

September 1998

\section{Measurement and Control Systems \\ Internal Report, 98 I/04}

Paper submitted to the American Control Conference, 1999

Eindhoven, September, 1998 


\title{
On Finite-Time Control of Inner Systems
}

\author{
J.H.A. Ludlage \\ Department of Electrical Engineering \\ Eindhoven University of Technology, The Netherlands, \\ j.h.a.ludlage@ele.tue.nl \\ S. Weiland \\ Department of Electrical Engineering \\ Eindhoven University of Technology, The Netherlands \\ s.weiland@ele.tue.nl \\ A.A. Stoorvogel \\ Department of Mathematics \\ Eindhoven University of Technology, The Netherlands \\ wscoas@win.tue.nl
}

\begin{abstract}
It is well known that non-minimum phase zeros of dynamical systems restrict the achievable performance of closed-loop transfer functions. If performance is specified in terms of finite time properties, then it is not evident that the zero structure of transfer functions limits the achievable performance of the controlled system. In this paper it is shown that the Hankel singular values of the inner factor of the system transfer function provide relevant information for achievable (finite time) performance of the controlled process.
\end{abstract}

\section{Introduction}

Since the beginning of the development of control theory it has been recognized $[4,19,27]$ that the process behavior limits the achievable performance of the controlled system. It is therefore important to have a detailed understanding of which characteristics of a process limit achievable performance of the controlled unit $[1,6,14,20,21]$ and how these characteristics effect the controller design $[4,13,15,17,19$, $22,27]$.

In the literature, various definitions and concepts have been proposed to formalize the influence of the open-loop process on achievable closed-loop behavior. Typical notions include controllability $[2,19,22,27]$, reproducibility [4], dynamic operability [1], dynamic resilience [15,21]. Most of these definitions are not very precise, but they express how the process puts limitations on the achievable closed loop behavior, independent of the controller.

It is well known that non-minimum phase plants are difficult to control and that non-minimum phase zeros put intrinsic limitations on achievable closed loop performance. More specifically, the relation between non-minimum phase zeros and closed-loop behavior is described by complex integral relations $[3,5,8,9]$ and the theory of $H_{\infty}$ optimal con- trol brought a clear understanding of the fact that for nonminimum phase and strictly proper systems the sensitivity function $\left|S\left(e^{j \phi}\right)\right|$ can be made less than one for certain frequencies $\phi$ only if it is larger than one for others [7]. Therefore, desirable properties of the sensitivity function in one frequency range have to be traded-off against undesirable behavior at other frequencies $[8,9]$.

Various approaches exist towards the analysis of controllability properties of non-minimum phase systems. The integral relations provide insight in the controllability of the system only for relatively simple cases. In more complex cases these relations provide little understanding of the intrinsic limitations of non-minimum phase zeros for control design. A second and frequently applied approach is what is called standardization of the problem. In most cases, this means that an inner-outer factorization is applied to the transfer function of the plant and the complementary sensitivity is chosen equal to the inner factor $[10,11,22]$. The influence of non-minimum phase zeros on the closed loop performance is then measured by considering the behavior of the corresponding sensitivity matrix viewed as function of frequency. A second type of standardization is analog to the Wolovich and Falb interactor matrix [26] and amounts to choosing the sensitivity matrix $S$ triangular [16,23,24]. In this case it is assumed that the outputs are ordered in a descending order of importance; output one has absolute priority above output two and so on. As a result, the influence of non-minimum phase zeros on the outputs of the closed loop is shifted towards the lowest priority outputs. In many cases such an approach results in unrealistic behavior at the process input and at the lowest priority outputs. From an analysis point of view the key disadvantage of standardization procedures is that the freedom in the control design is not entirely used. It therefore does not provide insight and understanding on how the process behavior actually limits the controlled process behavior. 
In this paper a new framework is developed to analyze the restrictions that non-minimum phase zeros pose on the controlled behavior. We will base this analysis on a study of finite time properties of the controlled system. It is shown that the Hankel singular values of the inner factor of a transfer function provide crucial information on the controllability of a dynamical system. The theory is illustrated by the solutions of a tracking and a deadbeat control problem.

The paper is organized as follows. In Section 2 preliminary definitions and results are given. Two control problems are formalized in Section 3. Section 4 provides the main theoretical results which are the basis for the solutions of the problems formulated in Section 3. Section 5 provides complete solutions to the control problems of Section 3 and discusses its consequences. Conclusions are deferred to Section 6.

\section{Notation and preliminaries}

Let $\mathbb{Z}$ denote the set of integers, $T \subseteq \mathbb{Z}, q \geq 1$ and define the function class

$$
\ell_{2}\left(T, \mathbb{R}^{q}\right):=\left\{x:\left.T \rightarrow \mathbb{R}^{q}\left|\|x\|_{2}^{2}:=\sum_{t \in T}\right| x(t)\right|^{2}<\infty\right\}
$$

where $|x(t)|$ is the standard Euclidean norm of $x(t)$. $\ell_{2}\left(T, \mathbb{R}^{q}\right)$ is equipped with the standard inner product $\langle x, y\rangle:=\sum_{t \in T} y^{T}(t) x(t) . \mathbb{Z}_{-}$and $\mathbb{Z}_{+}$denote the negative and the non-negative elements of $\mathbb{Z}$, respectively. Whenever the signal space is clear from the context we use the shorthand $\ell_{2}(T)$ for $\ell_{2}\left(\mathbb{Z}, \mathbb{R}^{q}\right)$. The shift operator, $s$, maps $T \subset \mathbb{Z}$ to the interval $s T:=\{t+1 \mid t \in T\}$. The support of a signal $u$ is defined as $\operatorname{supp}(u):=\{t \in \mathbb{Z} \mid u(t) \neq 0\}$. $\mathscr{R} \mathscr{H}_{\infty}^{p \times m}$ will be the class of all $p \times m$ stable proper rational transfer matrices. A transfer function $G \in \mathcal{R} \mathscr{H}_{\infty}^{p \times m}$ is identified with the state space system

$$
\begin{aligned}
x(t+1) & =A x(t)+B u(t) \\
y(t) & =C x(t)+D u(t)
\end{aligned}
$$

and the convolution system

$$
y(t)=\sum_{k=-\infty}^{\infty} g(t-k) u(k)
$$

whenever $g$ is the inverse Laplace transform of $G$ and $G(z)=C(z I-A)^{-1} B+D$. Throughout it is assumed that $(A, B, C, D)$ defines a minimal realization of $G$. Zeros and poles of $G$ are the roots of the numerator and denominator polynomials, respectively, of the Smith-McMillan form of $G$ (See [12]). A complex number $\lambda \in \mathbb{C}$ is a non-minimum phase zero if it is a zero of $G$ with $|\lambda| \geq 1$, it is called a minimum phase zero if it is a zero with $|\lambda|<1$.

Let $T_{u}$ and $T_{y}$ be two intervals ${ }^{1}$ of $\mathbb{Z}$. The observability

\footnotetext{
${ }^{1}$ An interval of $\mathbb{Z}$ is the set of all integers $t \in \mathbb{Z}$ with $a<t<b$ where $a, b \in \mathbb{Z} \cup\{\infty,-\infty\}$. An interval is called finite if $a, b \in \mathbb{Z}$, it is infinite if either $a=-\infty$ or $b=\infty$. It is empty if $a>b$.
}

and controllability gramian associated with (1) are given by

$$
\begin{aligned}
& Q\left(T_{y}\right):=\sum_{t \in T_{y}}\left(A^{T}\right)^{t} C^{T} C A^{t}, \\
& P\left(T_{u}\right):=\sum_{t \in s T_{u}} A^{-t} B B^{T}\left(A^{T}\right)^{-t} .
\end{aligned}
$$

The (possibly infinite) observability and controllability matrix are

$$
\begin{aligned}
& \mathcal{O}\left(T_{y}\right):=\operatorname{col}\left(C A^{t}, t \in T_{y}\right), \\
& \mathcal{C}\left(T_{u}\right):=\operatorname{row}\left(A^{-t} B, t \in s T_{u}\right),
\end{aligned}
$$

where "col" means stacking subsequent entries (lowest indexed entry on top) and "row" means placing subsequent entries side by side (lowest indexed entry on left). The gramians are well defined if either $T_{u} \subseteq \mathbb{Z}_{-}$and $T_{y} \subseteq \mathbb{Z}_{+}$, or $A$ is invertible and $T_{y}$ is left-bounded and $T_{u}$ is rightbounded. We further define the gramians $Q:=Q\left(\mathbb{Z}_{+}\right)$and $P:=P\left(\mathbb{Z}_{-}\right)$. The state space system (1) is called input balanced, output balanced or input-output balanced if both $P$ and $Q$ are diagonal and, respectively, $P=I, Q=I$ or $P=Q$.

We will be interested in the convolution system (2) where the time instants of the outputs are restricted to $T_{y}$ and the inputs are assumed to have support in $T_{u}$, i.e., we consider (2) for $t \in T_{y}$ and for input signals $u \in \ell_{2}$ with support supp $u \subseteq T_{u}$. Clearly, this defines an operator

$$
\Psi_{T_{u}, T_{y}}: \ell_{2}\left(T_{u}, \mathbb{R}^{m}\right) \rightarrow \ell_{2}\left(T_{y}, \mathbb{R}^{p}\right) .
$$

$\Psi_{T_{u}, T_{y}}$ is called a Hankel operator if $\max T_{u}+1 \leq \min T_{y}$, it is called a Toeplitz operator if $\min T_{u}=\min T_{y}$. Let

$$
\left\|\Psi_{T_{u}, T_{y}}\right\|:=\sup _{u \in \ell_{2}\left(T_{u}, \mathbb{R}^{m}\right)} \frac{\left\|\Psi_{T_{u}, T_{y}} u\right\|_{2}}{\|u\|_{2}}
$$

be the induced norm of $\Psi_{T_{u}, T_{y}}$. We call this norm a Hankel norm (Toeplitz norm) if $\Psi_{T_{u}, T_{y}}$ is Hankel (Toeplitz). Furthermore, the singular values $\sigma_{i}\left(\Psi_{T_{u}, T_{y}}\right)$ are assumed to be ordered according to $\sigma_{1} \geq \sigma_{2} \geq \ldots$ and are called Hankel or Toeplitz singular values whenever $\Psi_{T_{u}, T_{y}}$ is Hankel or Toeplitz. The Hankel singular values associated with $G$ are, by definition, the singular values of the Hankel operator $\Psi_{\mathbb{Z}_{-}, \mathbb{Z}_{+}}$. The following lemma summarizes some basic properties.

Lemma 2.1 Suppose that the intervals $T_{u}$ and $T_{y}$ are such that $Q\left(T_{y}\right)$ and $P\left(T_{u}\right)$ are well defined. If $\Psi_{T_{u}, T_{y}}$ is Hankel then $\Psi_{T_{u}, T_{y}}=\mathcal{O}\left(T_{y}\right) \mathcal{C}\left(T_{u}\right)$ and $\left\|\Psi_{T_{u}, T_{y}}\right\|=$ $\lambda_{\max }^{1 / 2}\left(P\left(T_{u}\right) Q\left(T_{y}\right)\right)$. Furthermore, $\Psi_{\mathbb{Z}_{+}, \mathbb{Z}_{+}}$is Toeplitz and satisfies $\left\|\Psi_{\mathbb{Z}_{+}, \mathbb{Z}_{+}}\right\|=\|G\|_{\infty}$.

The transfer function $G \in \mathcal{R H}_{\infty}^{p \times m}$ is called inner if it is norm preserving, i.e. $\|G u\|_{2}=\|u\|_{2}$ for all $u \in L_{2}$. An inner transfer function $G \in \mathcal{R} \mathcal{H}_{\infty}^{p \times m}$ is called minimum phase inner if it has no zeros. $G \in \mathcal{R} \mathcal{H}_{\infty}^{p \times p}$ is called outer if its inverse is stable, i.e. $G^{-1} \in \mathcal{R} \mathcal{H}_{\infty}^{p \times p}$. It has been shown 
in [25] that a transfer matrix $G \in \mathcal{R} \mathcal{H}_{\infty}^{p \times m}$, with no poles and zeros on the unit circle and $p \geq m$ admits an innerouter factorization $G=G_{\mathrm{i}} G_{0}$ with $G_{\mathrm{i}} \in \mathcal{R} \mathcal{H}_{\infty}^{p \times m}$ inner and $G_{0} \in \mathcal{R} \mathcal{H}_{\infty}^{m \times m}$ outer. The following result shows that the number of zeros of an inner system is directly related to the number of Hankel singular values which are equal to one.

\section{Lemma 2.2 Let $G \in \mathcal{R} \mathcal{H}_{\infty}^{p \times m}$ be inner. Then}

1. $p \geq m$ and all its Hankel singular values are smaller or equal to one.

2. If $p=m$ then all Hankel singular values equal one.

3. All zeros of $G$ are non-minimum phase zeros.

4. The zeros of $G$ are a subset of the inverse of the eigenvalues of $A$ and the number of zeros equals the number of Hankel singular values equal to one.

\section{Problem formulation}

In this section we formalize two control problems that involve the tracking of a reference signal on a finite time interval.

Let $G \in \mathcal{R H}_{\infty}$ be an inner square system with $\lim _{|z| \rightarrow \infty} G(z)$ invertible. Let $N>0$ and define the time intervals $T_{u}=[-N,-1], T_{y}=[0, \infty)$ and suppose that a reference signal $y_{\text {ref }} \in \ell_{2}$ is given with support $\operatorname{supp}\left(y_{\text {ref }}\right) \subseteq T_{u} \cup T_{y}$. Consider the following tracking problem.

Definition 3.1 (Tracking problem) The finite time tracking problem amounts to finding a control $u \in \ell_{2}\left(T_{u}\right)$ such that

$$
y_{\mathrm{ref}} \mid T_{u}=\Psi_{T_{u}, T_{u}} u
$$

Moreover, we wish to find a recursive expression for updating the control $u$ as a function of the length $N$ of the control horizon $T_{u}$ and characterize the resulting increase in control effort.

Note that this problem is in fact equivalent to the construction of the inverse of the Toeplitz operator $\Psi_{T_{u}, T_{u}}$. By invertibility of $\lim _{|z| \rightarrow \infty} G(z)$ this inverse exist so that the control $u$ exists and is unique. In the next section we will investigate the invertibility of Toeplitz operators and in Section 5 we will provide a solution of this recursive tracking problem and discuss its consequences. In particular, we will be interested in the effect of the controls $u$ on the output $y(t)$ of the system for time $t \geq 0$ as a function of the control horizon $N$.

The second control problem will be a finite time version of the well known deadbeat control problem. Specifically, let $G \in \mathcal{R} \mathscr{H}_{\infty}$ be an inner square system. Let $N>0$ and consider the intervals $T_{u}=[-N,-1], T_{y}=[0, \infty)$ and
$T_{u y}=T_{u} \cup T_{y}$. As before, let $y_{\text {ref }} \in \ell_{2}$ be a reference signal, with supp $y_{\text {ref }} \subseteq T_{u y}$. The basic idea of deadbeat is that the output of the system equals the reference signal after a predefined number of samples, say $n_{\mathrm{db}}$. Since the control horizon $T_{u}$ is finite it is, in general, not possible to track an arbitrary reference signal on the infinite time interval $T_{y}$. We therefore consider the problem of finding $u \in \ell_{2}$ with $\operatorname{supp}(u) \subseteq T_{u}$ such that the output $y$ tracks $y_{\text {ref }}$ on a finite interval. Let $0<n_{\mathrm{db}}<N$ and define the interval $T_{u}^{\prime}=\left[-N+n_{\mathrm{db}},-1\right]$

Definition 3.2 (Deadbeat control problem) The deadbeat control problem amounts to finding $u \in \ell_{2}\left(T_{u}\right)$ such that

$$
\begin{cases}y_{\mathrm{ref}} \mid T_{u}^{\prime}=\Psi_{T_{u}, T_{u}^{\prime} u} & \text { and } \\ \left\|\Psi_{T_{u}, T_{y}} u-y_{\mathrm{ref}} \mid T_{y}\right\|_{2} & \text { is minimal. }\end{cases}
$$

In addition we will be interested in finding the minimal $n_{\mathrm{db}}$ such that this problem is solvable for all $y_{\text {ref }} \in \ell_{2}\left(T_{u y}\right.$.

\section{Inner systems on a finite time horizon}

In this section the structural properties of the Toeplitz operator $\Psi_{T_{u}, T_{u}}$ associated with an inner square system $G$ and an arbitrary (finite) interval $T_{u}$ will be studied. An elegant expression for the singular value decomposition of $\Psi_{T_{u}, T_{u}}$ for an inner square system is given as follows.

Theorem 4.1 Let $G \in \mathscr{R}_{\infty}^{m \times m}$ be inner with minimal input-output balanced realization $(A, B, C, D)$ and McMillan degree $n$. Let $T_{u}$ be a finite interval of length $N$ and let $T_{y}:=\left\{t \in \mathbb{Z} \mid t>\max \left(T_{u}\right)\right\}$. Then

1. $m N-n$ singular values of $\Psi_{T_{u}, T_{u}}$ equal one.

2. The $n$ smallest singular values of $\Psi_{T_{u}, T_{u}}$ equal the singular values of $A^{N}$.

3. The span of the right singular vectors of $\Psi_{T_{u}, T_{u}}$ corresponding to its smallest $n$ singular values equals $\operatorname{im} \mathcal{C}\left(T_{u}\right)^{T}$.

4. The span of the left singular vectors of $\Psi_{T_{u}, T_{u}}$ corresponding to its smallest $n$ singular values equals $\operatorname{im} \mathcal{O}\left(-s T_{u}\right)$.

Moreover, for any $u \in \ell_{2}$ with $\operatorname{supp} u \subseteq T_{u}$ and $\underline{u}:=$ $\operatorname{col}\left(u(t), t \in T_{u}\right) \in \operatorname{im} \mathcal{C}\left(T_{u}\right)^{T}$

$$
\begin{aligned}
& \Psi_{T_{u}, T_{u}} u=-\mathcal{O}\left(-s T_{u}\right)\left(A^{T}\right)^{N} P\left(T_{u}\right)^{-1} \mathcal{C}\left(T_{u}\right) \underline{u} \\
& \Psi_{T_{u}, T_{y}} u=\mathcal{O}\left(T_{y}\right) \mathcal{C}\left(T_{u}\right) \underline{u}
\end{aligned}
$$

We remark that a similar result holds for non-square inner functions. From Theorem 4.1 we infer that the closer the singular values of the Hankel operator $\Psi_{T_{u}, T_{y}}$ are to one the closer the singular values of the Toeplitz $\Psi_{T_{u}, T_{u}}$ are to zero. The $n$ smallest singular values of the Toeplitz operator are decreasing functions of $N$ that converge to zero for $N \rightarrow \infty$. 
This observation has important consequences for control. Indeed, if a reference signal $y_{\text {ref }} \in \ell_{2}\left(T_{u}\right)$ needs to be tracked by choosing a suitable control $u \in \ell_{2}\left(T_{u}\right)$, then

$$
u=\Psi_{T_{u}, T_{u}}^{\dagger} y_{\mathrm{ref}}
$$

with $\dagger$ denoting the Moore-Penrose inverse, defines the control with minimal $\ell_{2}$ norm that achieves tracking. By Theorem $4.1,\|u\|_{2}$ will be large unless $\operatorname{col}\left(u(t), t \in T_{u}\right)$ is orthogonal to the left singular vectors associated with the $n$ smallest singular values of $\Psi_{T_{u}, T_{u}}$. This control will moreover achieve that the output $y$ on the interval $T_{y}$ equals

$$
y:=\Psi_{T_{u}, T_{y}} u=\Psi_{T_{u}, T_{y}} \Psi_{T_{u}, T_{u}}^{\dagger} y_{\mathrm{ref}}
$$

which has large norm $\|y\|_{2}$, as the right singular vectors corresponding to the $n$ smallest singular values of $\Psi_{T_{u}, T_{u}}$ exactly align with the right singular vectors corresponding to the $n$ nonzero singular values of $\Psi_{T_{u}, T_{y}}$. Statement 2 of Theorem 4.1 moreover shows that this effect worsens if the length of $T_{u}$ is increased. Hence, since the Hankel singular values of $\Psi_{T_{u}, T_{y}}$ converge to one (as $N \rightarrow \infty$ ), tracking problems with norm constraint inputs or norm constraint (future) outputs can only be solved on relatively small control intervals $T_{u}$. The maximum length of these intervals is determined by the convergence rate to this limit, which is determined by the eigenvalues of $A$.

\section{Main results}

\subsection{The tracking problem}

Consider the tracking problem formulated in Section 2, i.e., let $N>0$ and define $T_{u}=[-N,-1], T_{u}^{\prime}=[-N, 0]$, $T_{y}=[0, \infty)$ and $y_{\text {ref }} \in \ell_{2}$ be a reference signal with support $\operatorname{supp}\left(y_{\mathrm{ref}}\right) \subseteq T_{u} \cup T_{y}$. Let $\Pi_{\mathcal{O}}$ be the orthogonal projector onto $\operatorname{im} \mathcal{O}\left(-s T_{u}\right)$, i.e.,

$$
\Pi_{\mathcal{O}}=\mathcal{O}\left(-s T_{u}\right) Q^{-1}\left(-s T_{u}\right) \mathcal{O}\left(-s T_{u}\right)^{T}
$$

and $\Pi_{\mathcal{O}^{\perp}}=I-\Pi_{\mathcal{O}}$ the projection on its orthogonal complement. Let

$$
\begin{aligned}
& y_{\text {ref } 1}=\Pi_{\mathcal{O}^{\perp}} y_{\text {ref }} \mid T_{u} \\
& y_{\text {ref } 2}=\Pi_{\mathcal{O}} y_{\text {ref }} \mid T_{u}
\end{aligned}
$$

Then $y_{\text {ref }} \mid T_{u}=y_{\text {ref1 }}+y_{\text {ref2 }}$ and $\left\langle y_{\text {ref1 } 1}, y_{\text {ref2 } 2}\right\rangle=0$. Decompose the input as

$$
u=u_{1}+u_{2}
$$

where $u_{1}$ is such that $y_{\mathrm{ref} 1}=\Psi_{T_{u}, T_{u}} u_{1}$ and $u_{2}$ is such that $y_{\text {ref2 }}=\Psi_{T_{u}, T_{u}} u_{2}$. From Theorem 4.1 we obtain that $\left\langle u_{1}, u_{2}\right\rangle=0$ and the following result can be derived.

\section{Theorem 5.1 Under the above conditions}

$$
\text { - }\left\|u_{1}\right\|_{2}=\left\|y_{\text {ref } 1}\right\|_{2}
$$

- $u_{1}$ does not effect the output of the system on the time interval $T_{y}$, i.e., $\Psi_{T_{u}}, T_{y} u_{1}=0$.

- Denote the orthogonal complement of $\mathcal{C}\left(T_{u}\right)$ and $\mathcal{O}\left(-s T_{u}\right)$ as $\mathcal{C}\left(T_{u}\right)^{\perp}$ and $\mathcal{O}\left(-s T_{u}\right)^{\perp}$, then the control (4) solves the tracking problem if

$$
\begin{gathered}
\operatorname{col}\left(u_{1}(t), t \in T_{u}\right)=\left(\mathcal{C}\left(T_{u}\right)^{\perp}\right)^{T}\left(P\left(T_{u}\right)^{\perp}\right)^{-1 / 2} \\
\left(Q\left(-s T_{u}\right)^{\perp}\right)^{-1 / 2}\left(\mathcal{O}\left(-s T_{u}\right)^{\perp}\right)^{T} y_{\text {ref } 2} \\
\operatorname{col}\left(u_{2}(t), t \in T_{u}\right)=-\mathcal{C}\left(T_{u}\right)^{T} P\left(T_{u}\right)^{-1}\left(A^{T}\right)^{-N} \\
\mathcal{O}\left(-s T_{u}\right)^{T} y_{\text {ref } 2}
\end{gathered}
$$

- The control $u^{\prime}$ in the tracking problem is a function of $u$ and $y_{\text {ref }}$ defined as

$$
u^{\prime}(t)=\left\{\begin{array}{lc}
u(t) & \text { if } t \in T_{u} \\
D^{-1} y_{\text {ref }}(0)+D^{-1} C\left(A^{T}\right)^{-N} \mathcal{O}\left(-s T_{u}\right)^{T} y_{\text {ref } 2} & \text { if } t=0
\end{array}\right.
$$

Observe that the second term on the right hand side of (5) exactly compensates the influence of $u$ on the output at time 0 . Moreover, whether $\left\|u^{\prime}(0)\right\|$ diverges or not, for increasing $N$, is determined by this second term and hence by the alignment of $y_{\mathrm{ref}} \mid T_{u}$ to $\mathcal{O}\left(-s T_{u}\right)$. Hence a necessary condition for $u^{\prime}(0)$ to converge for $N \rightarrow \infty$ is that $\mathcal{O}\left(-s T_{u}\right)^{T} y_{\text {ref } 2} \rightarrow 0$. Equivalently, on the infinite horizon convergence is achieved only if $y_{\text {ref }} \perp \mathcal{O}$.

Let $e=y_{\mathrm{ref}} \mid T_{y}-\Psi_{T_{u}, T_{y}} u$ and $e^{\prime}=y_{\mathrm{ref}} \mid T_{y}-\Psi_{T_{u}^{\prime}, T_{y}} u^{\prime}$ then $e$ and $e^{\prime}$ are the tracking error signals over the horizon $T_{y}$, due to the control actions $u$ and $u^{\prime}$, respectively. We can actually derive that

$$
\begin{aligned}
& e^{\prime}(0)=0 \\
& \left\|e^{\prime}\right\|_{\ell_{2}\left(T_{y}^{\prime}\right)} \geq\|e\|_{\ell_{2}\left(T_{y}^{\prime}\right)}
\end{aligned}
$$

Hence the control action $u^{\prime}(t)$ at time $t=0$ achieves instantaneous tracking of the reference signal $y_{\mathrm{ref}}(0)$ at the cost of a non-decreasing norm of the future tracking error, i.e. it has an opposite effect on the error on the horizon $T_{y}^{\prime}$. For a receding horizon real time implementation of the control actions, this means that subsequent updates of the control actions $u^{\prime}(0)$ result in magnitudes $\left\|u(0)^{\prime}\right\|$ that diverge at a rate corresponding to the inverse of the eigenvalues of $A^{N}$, where $N$ is the (receding) horizon.

\subsection{The deadbeat control problem}

Consider the deadbeat control problem formalized in Section 2. That is, let $T_{u}=[-N,-1], T_{y}=[0, \infty)$ and $T_{u y}=T_{u} \cup T_{y}$ and let $y_{\text {ref }} \in \ell_{2}$ be a reference signal, with supp $y_{\text {ref }} \subseteq T_{u y}$. For $0<n_{\mathrm{db}}<N$ we introduce $T_{0}=\left[-N,-N+n_{\mathrm{db}}-1\right]$ and $T_{u}^{\prime}=\left[-N+n_{\mathrm{db}},-1\right]$. The following result provides necessary and sufficient conditions for its solvability. 
Theorem 5.2 Let $T_{I}=s^{\left(N-n_{d b}\right)} T_{0}$. Then the deadbeat control problem is solvable if and only if the Gramian $P\left(T_{I}\right)$ is non-singular. In this case, a solution is given by the control

$$
u(t)=u_{1}(t)+u_{2}(t) ; \quad t \in T_{u}
$$

where

$$
\left\{\begin{aligned}
\operatorname{col}\left(u_{1}(t), t \in T_{u}\right)= & \operatorname{col}\left(0,\left[\Psi_{T_{u}^{\prime}, T_{u}^{\prime}}\right]^{\dagger} y_{r e f 1}^{\prime}\right) \\
\operatorname{col}\left(u_{2}(t), t \in T_{u}\right)= & \operatorname{col}\left(\mathcal{C}\left(T_{I}\right)^{T} P\left(T_{I}\right)^{-1} A^{n_{d b}} Q^{-1}\right. \\
& \left.\mathcal{O}^{T} y_{r e f 2}^{\prime}, 0\right)
\end{aligned}\right.
$$

Here, $y_{\text {refl }}=\Pi_{\mathcal{O}^{\perp}} y_{\text {ref }}, y_{\text {ref } 2}=\Pi_{\mathcal{O}} y_{\text {ref }}, \quad y_{\text {refl }}^{\prime}=$ $\operatorname{col}\left(y_{\text {refl }} ; t \in T_{u}^{\prime}\right), y_{\text {ref } 2}^{\prime}=\operatorname{col}\left(y_{\text {ref2 }} ; t \in T_{u}^{\prime}\right)$,

From Theorem 5.2 we obtain that the existence of a solution to the deadbeat control problem is determined by the regularity of $P\left(T_{I}\right)$, i.e. at the moment that all states can be observed at the output. It is well known that this condition is fulfilled if $n_{d b} \geq n$. The minimum value of $n_{\mathrm{db}}$ for which $P\left(T_{I}\right)$ is non-singular, say $n_{\min }$, may therefore be smaller than $n$.

A further consequence of Theorem 5.2 is that for $n_{d b}>$ $n_{\min }$ the solution of the deadbeat control problem is not necessarily unique. In this case $\left.u\right|_{T_{0}}$ is not unique, since rank $\mathcal{C}\left(T_{O}\right)^{\perp}=n_{d b}-n_{\min }$. Hence any solution will be of the form

$\operatorname{col}\left(u(t) ; t \in T_{O}\right)=\mathcal{C}\left(T_{O}\right)^{\perp} z+\mathcal{C}\left(T_{O}\right)^{T} P\left(T_{I}\right)^{-1} A^{n_{d b}} X_{2}\left(T_{u^{\prime} y}\right)$

with $z \in \mathbb{R}^{n_{d b}-n_{\min }}$, solves problem 2 . Hence $z$ exactly parameterizes the non-uniqueness of the solution of DBP. This freedom can still be used e.g. to reduce the tracking error on the interval $\left[-N+n_{\min },-N+n_{d b}\right]$.

\section{Example}

As an illustration consider the transfer function

$$
G(z)=-0.3136 \frac{(z-1.0204)(z-1.25)(z-2.5)}{(z-0.98)(z-0.8)(z-0.4)} .
$$

Then $G$ is square inner and has three non-minimum phase zeros. Let $T_{u}=[1, N]$. The three smallest singular values of the Toeplitz operator $\Psi_{T_{u}, T_{u}}$ associated with $G$ are depicted, as functions of $N$, in figure 1 for $N=3, \ldots, 200$. For $N=100$, the corresponding left and right singular vectors are depicted in figure 2 . The right singular vectors corresponding to the three smallest singular values increase as function of time. Now, consider the deadbeat problem with reference signal $y_{\text {ref }} \mid T_{u y}=\mathcal{O}\left(-s T_{u y}\right) w$, with $w=\left[\begin{array}{lll}-10.1813 & -1.5275 & 2.0837\end{array}\right]^{T}$, i.e. the steady state value of the states for a unit step. In figure 3 the influence of the choice of the deadbeat horizon $n_{\mathrm{db}}$ on $\left\|\left.y\right|_{T_{0}}\right\|_{2}$ and $\|u\|_{2}$ are given for $N=100$. These norms are decreasing functions of $n_{\mathrm{db}}$, i.e. the longer the deadbeat horizon, the less control effort is needed and the smaller the output-norm will be on the time interval $T_{0}$.

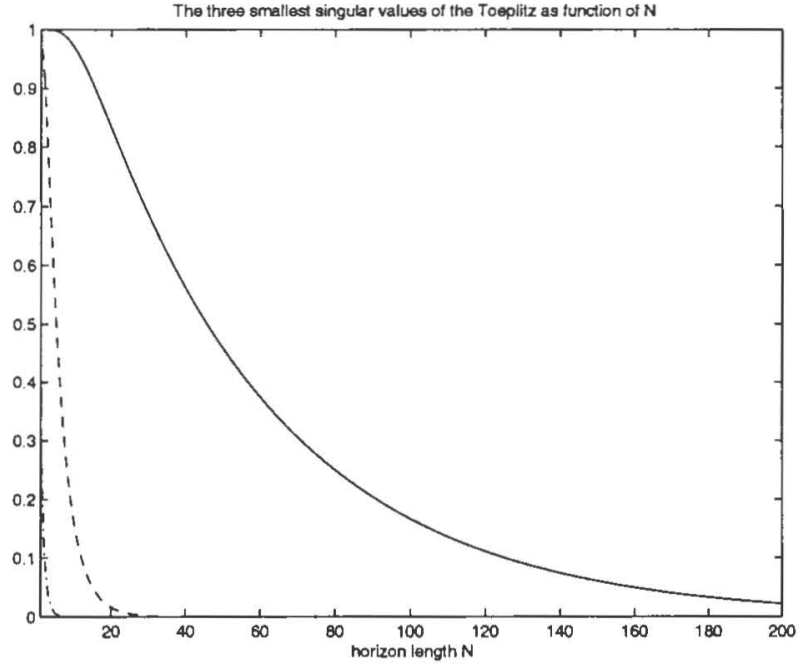

Figure 1: Singular values of $\Psi_{T_{u}, T_{u}}$ as function of $N$
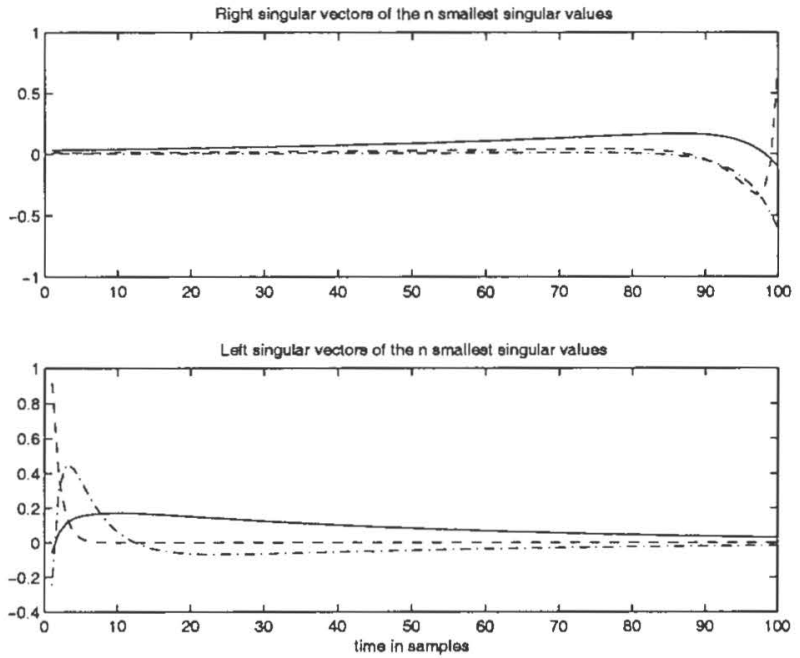

Figure 2: The right (upper figure) and left (lower figure) singular vectors corresponding to the three smallest singular values of $\Psi_{T_{u}, T_{u}}$

\section{Conclusions}

The finite time behavior of non-minimum phase zeros of square inner systems has been studied. The restrictions that non-minimum phase behavior poses on the controlled behavior of the system can be characterized by the limited invertibility of the operator from the $n$-dimensional subspace $\mathcal{C}^{T}\left(T_{u}\right)$ of the input subspace $\ell_{2}\left(T_{u}\right)$ to an $n$-dimensional output subspace $\mathcal{O}\left(-s T_{u}\right)$ of the output space $\ell_{2}\left(T_{u}\right)$. It is shown that this operator can only be invert over a restricted length $N$ of the control horizon $T_{u}$, since the inverse can only be obtained for increasing $N$ at the cost of a $\|u\|_{2}$ and a $\left\|\left.y\right|_{[0, \infty)}\right\|_{2}$ that both diverge as function of the inverse of the eigenvalues of $A$. It is shown that this behavior is due to the opposite effect that $u \in \operatorname{span} \mathcal{C}\left(T_{u}\right)$ has on $\left.y\right|_{[0, \infty]}$, compared to the behavior it invokes on $\left.y\right|_{T_{u}}$. It is thought by the authors that this characterization of the non-minimum 

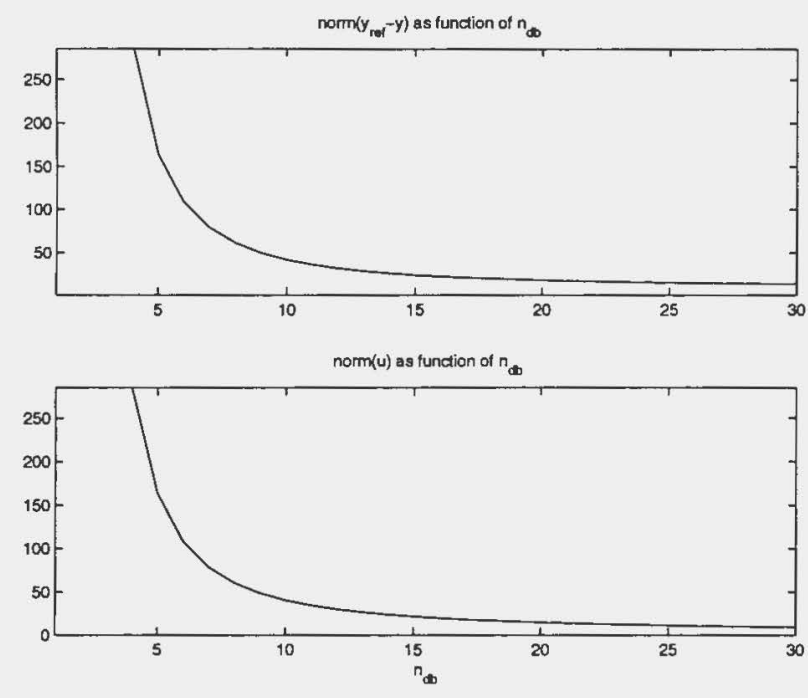

Figure 3: $\left\|\left.\left(y_{\text {ref }}-y\right)\right|_{T_{O}}\right\|_{2}$ (upper figure) and $\|u\|_{2}$ (lower figure) as function of the deadbeat horizon $n_{d b}$.

phase behavior is more elegant and gives clearer insight in the inherent restrictions that non-minimum phase puts on the controlled behavior then the commonly used infinite time characterizations. As an example of the application of the results found the deadbeat problem and nominal stability of receding horizon controllers have been discussed.

\section{References}

[1] Y. Arkun. Dynamic process operability. Import problems, recent results and new challenges. In: M. Morari and T. J. McAvoy, (Ed.), CACHE, Chem. Proc. Cont. (CPC III). Elsevier, 1986. pp.323-367.

[2] D. Biss and J.D. Perkins. Application of input-output controllability analysis to chemical processes. Proceedings of the Second European Control Conference ECC'93, Groningen, The Netherlands, june 28-july 2, 1993. pp.1056-1059.

[3] S.P. Boyd and C.A. Desoer. Subharmonic functions and performance bounds on linear time-invariant feedback systems. IMA J. Math. Cont. \& Info., Vol.2, 1985. pp.153-170.

[4] R.W. Brockett and M.D. Mesarovic. The reproducibility of multivariable systems. Journal of Mathematical Analysis and Applications, Vol.11, 1965. pp.548-563.

[5] J. Chen. Multivariable Gain Phase and Sensitivity integral relations and design tradeoffs IEEE Trans. on Automatic Control, AC-43, No.3, 1998. pp.373-385.

[6] S.Fararooy, J.D. Perkins, T.I. Malik, M.J. Oglesby and S. Williams. Process controllability toolbox (PCTB). Computers and Chemical Engineering Vol.17, No.5/6, 1993. pp.617-625.

[7] B.A. Francis and G. Zames. On optimal sensitivity theory for SISO feedback systems. IEEE Trans. on Automatic Control, AC-29, 1985.

[8] J.S. Freudenberg and D.P. Looze. Right half plane poles and zeros and design trade-offs in feedback systems. IEEE Trans. on Automatic Control, AC-30, No.6, 1985. pp.555-565.
[9] J.S. Freudenberg and D.P. Looze. Frequency Domain Properties of Scalar and Multivariable Feedback Systems. Lecture Notes in Control and Information Sciences, Vol.104, Springer Verlag, Berlin, 1988.

[10] B.R. Holt and M. Morari. Design of resilient processing plants V. The effect of deadtime on the dynamic resilience. Chemical Engineering Science, Vol.40, No.7, 1985. pp.1229-1237.

[11] B.R. Holt and M. Morari. Design of resilient processing plants VI. The effect of right half plane zeros on the dynamic resilience. Chemical Engineering Science, Vol.40, No.1, 1985. pp.59-74.

[12] T. Kailath. Linear Systems. Prentice-Hall Inc., Englewood Cliffs, N.J., 1980

[13] J.H.A. Ludlage. Controllability Analysis of Industrial Processes: Towards the industrial application. $\mathrm{PhD}$. thesis, Eindhoven University of Technology, Eindhoven, The Netherlands, 1997.

[14] T.E. Marlin and T.J. McAvoy. A short-cut method for process control and operability analysis. In M. Morari and T. J. McAvoy, (Eds.), CACHE, Chem. Proc. Cont. (CPC III). Elsevier, 1986. pp.369-419.

[15] M. Morari. Design of resilient processing plants III. A general framework for the assessment of dynamic resilience. Chemical Engineering Science, Vol.38, No.11, 1983. pp.1881-1891.

[16] M. Morari, E. Zafiriou and B.R. Holt. Design of resilient processing plants-X. New characterization of the effect of RHP zeros. Chemical Engineering Science, Vol.42, No.10, 1987. pp.24252428.

[17] M. Morari and E. Zafiriou. Robust Process Control. Prentice-Hall, 1989.

[18] J.B. Rawlings and K.R.Muske. The stability of constrained receding horizon control. IEEE Trans. on Automatic Control, AC38, No.10, 1993. pp.1512-1516.

[19] H.H. Rosenbrock. State space and Multivariable Theory. Academic Press, New York NY, USA, 1970.

[20] L.W. Russel and J.D. Perkins. Towards a method for diagnosis of controllability and operability problems in chemical plants. Chem. Eng. Res. Des., vol.65, 1987. pp.453-461.

[21] S. Skogestad, M. Hovd and P. Lundström. Simple frequency domain tools for analysis of inherent control limitations. Modeling, Identification and Control, Vol.12, No.4, 1991. pp.159-177

[22] S. Skogestad and I. Postlethwaite. Multivariable Feedback Control, Analysis and Design. John Wiley \& Sons Ltd, Chichester, UK, 1996.

[23] C.A. Tsiligiannis and S.A. Svoronos. Dynamic interactor in multivariable process control-I. The general time delay case. Chem. Eng. Science, Vol.43, No.2, 1988. pp.339-347.

[24] C.A. Tsiligiannis and S.A. Svoronos. Dynamic interactor in multivariable process control-II. Time delays and zeroes outside the unit circle. Chem. Eng. Science, Vol.44, No.9, 1989. pp.2041-2047.

[25] M. Vidyasagar. Control System Synthesis. A Factorization Approach. MIT press, 1985.

[26] W.A. Wolovich and P.L. Falb. Invariants and canonical forms under dynamic compensation. SIAM J. Control and Optimization, vol.14, No.6, 1976. pp.906-1008.

[27] J.G. Ziegler and N.B. Nichols. Process lags in automatic circuits. Transactions of the A.S.M.E. Vol.65, 1943. pp.433-444. 